\title{
Two-Month Angioscopic Evaluation of Superficial Femoral Artery Treated With Paclitaxel-Coated Nitinol Drug-Eluting Stent
}

Takayuki Ishihara, MD; Osamu Iida, MD; Masaki Awata, MD, PhD;

Masashi Fujita, MD, PhD; Tetsuya Watanabe, MD, PhD; Yoshio Ishida, MD, PhD; Shinsuke Nanto, MD, PhD; Masaaki Uematsu, MD, PhD
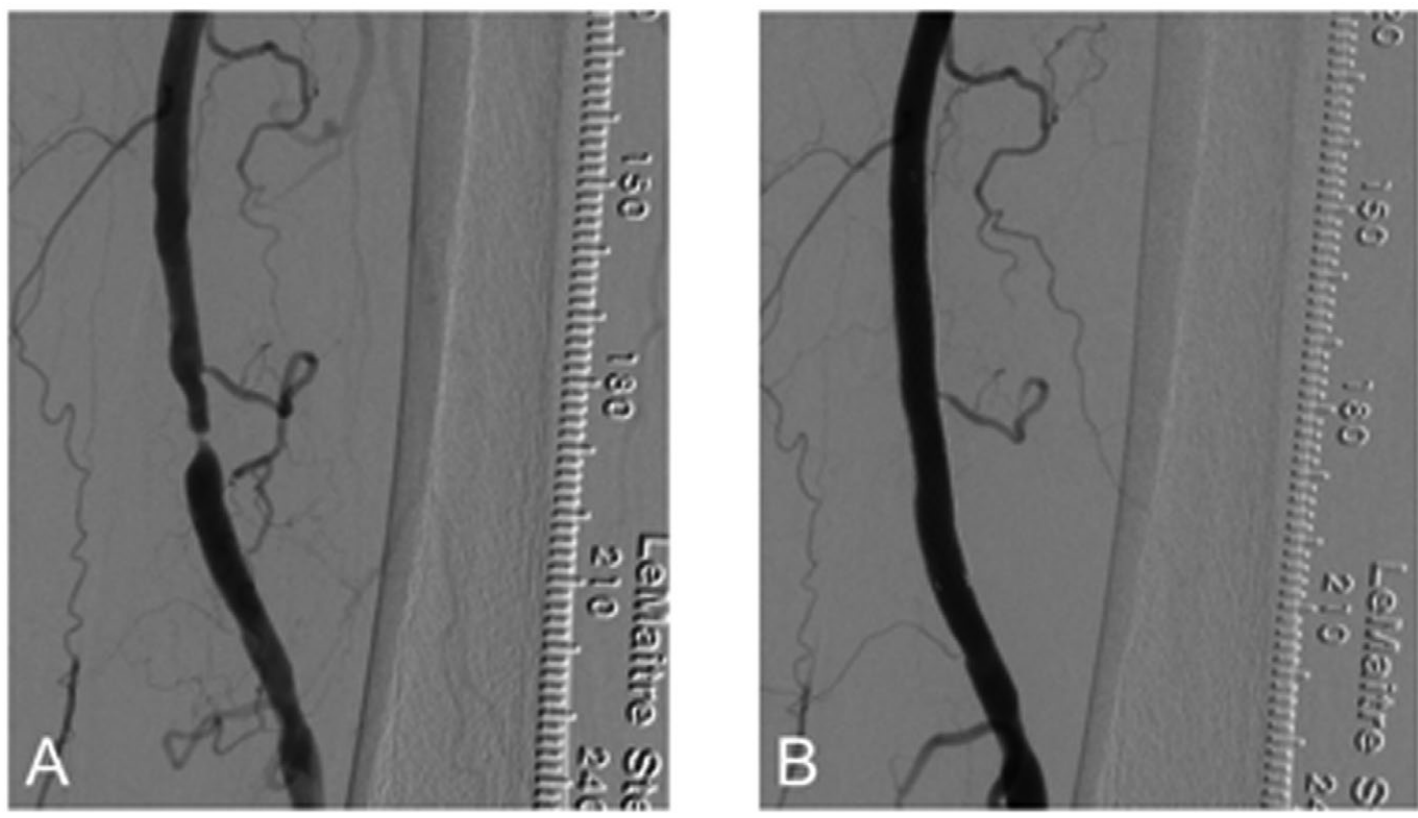

Figure 1. Angiography of left superficial artery before and after endovascular therapy. (A) Initial angiography showing severe stenosis in the left superficial femoral artery. (B) Final angiography showing good dilatation after implantation of a paclitaxelcoated nitinol drug-eluting stent (Zilver PTX; 7.0-mm diameter $\times 60 \mathrm{~mm}$ long), followed by post-dilatation using a 6.0-mm diameter $\times 40-\mathrm{mm}$ long balloon.

$\mathbf{P}$ olymer-free, paclitaxel-coated nitinol drug-eluting stent (Zilver PTX; Cook Medical, Bloomington, IN, USA) is now available for the treatment of peripheral artery disease in the superficial femoral artery (SFA), and durable clinical outcomes compared to the bare-metal stent counterpart have been reported. ${ }^{1-4}$ Arterial repair after Zilver PTX implantation in the SFA, however, remains uncharacterized.
A 76-year-old woman presented with life-limiting claudication in the left leg. Ankle brachial index (ABI) was 1.07/0.63 (right/left). Duplex ultrasound showed a decreased flow pattern of the left popliteal artery with normal flow pattern in the common femoral artery (CFA), and stenosis or occlusion of the left SFA was strongly suspected.

Angiography 1 month later showed severe stenosis in the

Received December 14, 2012; revised manuscript received January 28, 2013; accepted February 21, 2013; released online March 29, 2013 Time for primary review: 23 days

Kansai Rosai Hospital Cardiovascular Center, Amagasaki (T.I., O.I., M.A., M.F., T.W., Y.I., M.U.); Advanced Cardiovascular Therapeutics, Osaka University Graduate School of Medicine, Suita (S.N.), Japan

Name of grant: None.

Mailing address: Osamu Iida, MD, Kansai Rosai Hospital Cardiovascular Center, 3-1-69 Inabaso, Amagasaki 660-8511, Japan. E-mail: iida.osa@gmail.com

ISSN-1346-9843 doi:10.1253/circj.CJ-12-1538

All rights are reserved to the Japanese Circulation Society. For permissions, please e-mail: cj@j-circ.or.jp 


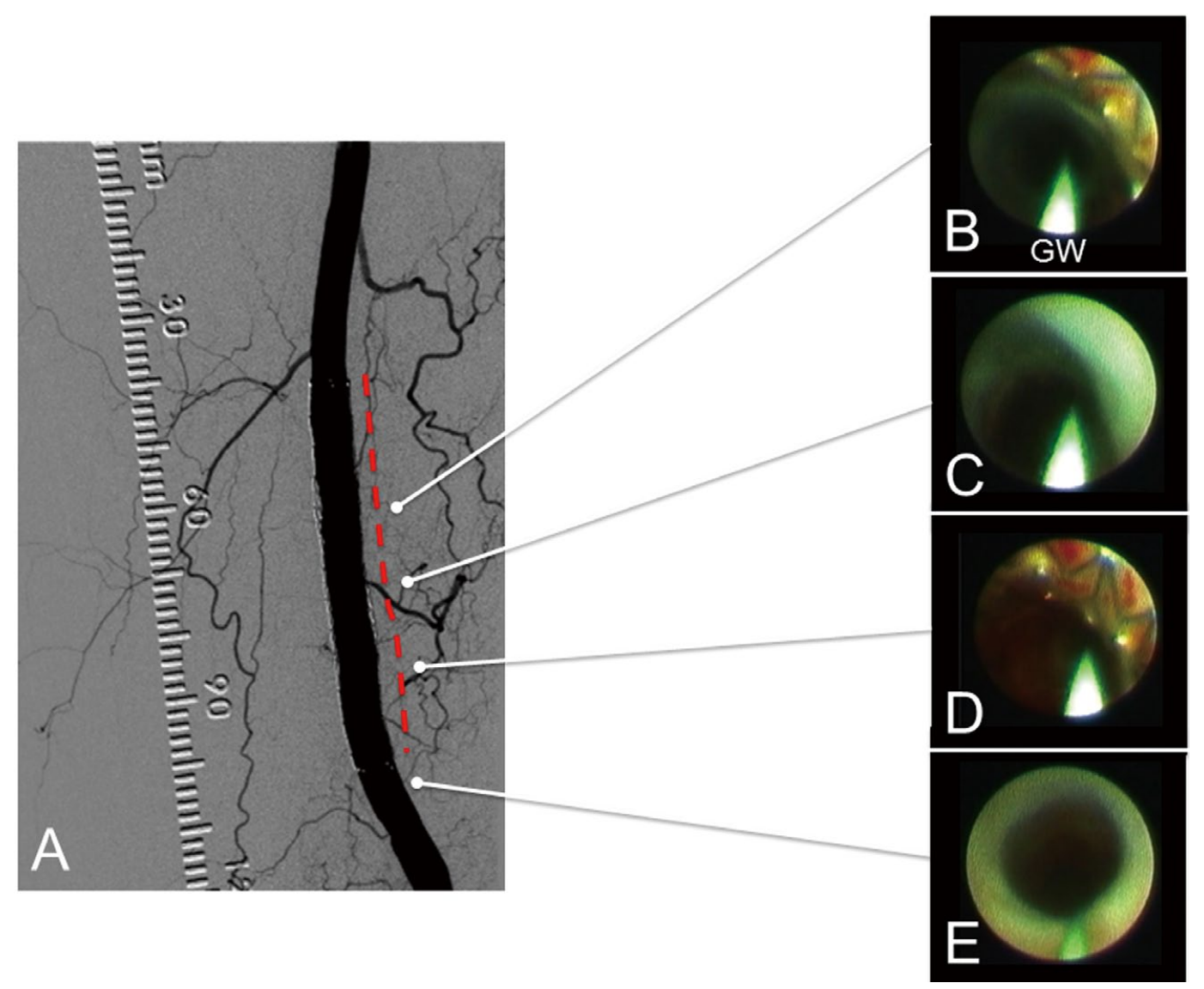

Figure 2. Angiography and angioscopy of intra-stent surfaces 2 months after paclitaxel-coated nitinol drug-eluting stent implantation. (A) Follow-up angiography 2 months after endovascular therapy showing a patent paclitaxel-coated nitinol drug-eluting stent. (B-E) Angioscopy showing (B,D) fully visible struts with red thrombus adhesion and yellow plaque underneath the stent in some areas, and (C) complete neointimal coverage without thrombus in other areas. (E) The wall color at the distal reference of the stented site was slightly yellow. Dotted red line, site of paclitaxel-coated nitinol drug-eluting stent implantation; GW, guidewire.

left SFA (Figure 1A), which was treated with endovascular therapy (EVT) after 2 weeks of dual anti-platelet therapy (DAPT) with aspirin $100 \mathrm{mg} /$ day and clopidogrel $75 \mathrm{mg} /$ day. Through a contralateral approach from the right CFA with a 6-Fr guiding sheath (ANSEL; Cook Medical) and following $5,000 \mathrm{U}$ of intra-arterial unfractionated heparin, the severe stenosis in the left SFA was crossed with a 0.014-in guidewire (Aguru Support; Boston Scientific, Natick, MA, USA). Vessel diameter was assessed on intravascular ultrasound (IVUS; Atlantic Pro SR2; Boston Scientific) and a Zilver PTX (7.0$\mathrm{mm}$ diameter $\times 60 \mathrm{~mm}$ long) was then was implanted followed by additional angioplasty (SHIDEN, $6.0-\mathrm{mm}$ diameter $\times 40 \mathrm{~mm}$ long; Kaneka, Osaka, Japan). Final angiogram showed good vessel patency (Figure 1B) with sufficient stent area as determined on IVUS. No perioperative complications occurred, and the patient was discharged 2 days after the procedure; she was completely free from quality-of-life-impairing claudication, and ABI of the left leg improved from 0.63 to 0.99 .

Follow-up angiography at 2 months after EVT indicated persistent patency of Zilver PTX (Figure 2A). Angioscopy (Vecmova NEO; FiberTech, Tokyo, Japan) showed fully visible struts with red thrombus adhesion and yellow plaque underneath the stent in some areas (Figures 2B,D), and com- plete neointimal coverage without thrombus in other areas (Figure 2C). The wall color at the distal reference of the stented site was slightly yellow (Figure $2 \mathrm{E}$ ). On the following day, the patient was discharged without any complication and DAPT was continued based on the angioscopy results.

Here, we report on angioscopic findings 2 months after Zilver PTX implantation in the SFA. Widely visible struts without neointima and with red thrombus were observed. To the best of our knowledge this is the first report on evaluation of intrastent status of Zilver PTX in the SFA.

Angioscopic evaluation 8 months after paclitaxel-eluting stent (PES) implantation in coronary arteries (TAXUS Express2; Boston Scientific) has been previously reported. ${ }^{5}$ Thrombus was observed in $43 \%$ of the stents and more frequently in PES than in sirolimus-eluting stents (Cypher; Cordis, Miami, FL, USA). Nakazawa et al reported on an autopsy evaluation of PES implanted in the coronary artery and suggested that etiology of late stent thrombosis after PES implantation might be associated with excessive fibrin deposition on the abluminal surface. ${ }^{6}$ In the present angioscopic evaluation, massive thrombus was apparent over a wide stented area. Because Zilver PTX has no polymer, paclitaxel itself seems to cause fibrin deposition leading to thrombus formation. 
Widely uncovered struts were observed 2 months after Zilver PTX implantation in the SFA. A ratio of uncovered struts to total stent struts per section of $>30 \%$ is a risk factor for stent thrombosis. ${ }^{7}$ The recommended duration of DAPT is 2 months because the paclitaxel elutes for 56 days. ${ }^{1}$ Although it remains unclear whether the present angioscopic finding is clinically relevant or not, the thrombogenicity observed might suggest the need for continuation of DAPT beyond 2 months after Zilver PTX implantation in the SFA.

We were able to perform angioscopic evaluation of an SFA treated with Zilver PTX 2 months after stent implantation. Widely visible struts without neointima and with red thrombus were observed, suggestive of thrombogenicity 2 months after Zilver PTX implantation.

\section{References}

1. Dake MD, Ansel GM, Jaff MR, Ohki T, Saxon RR, Smouse HB, et al; Zilver PTX Investigators. Paclitaxel-eluting stents show superiority to balloon angioplasty and bare metal stents in femoropopliteal disease: Twelve-month Zilver PTX randomized study results. Circ Cardiovasc Interv 2011; 4: 495-504.
2. Dake MD, Scheinert D, Tepe G, Tessarek J, Fanelli F, Bosiers M, et al; Zilver PTX Single-Arm Study Investigators. Nitinol stents with polymer-free paclitaxel coating for lesions in the superficial femoral and popliteal arteries above the knee: Twelve-month safety and effectiveness results from the Zilver PTX single-arm clinical study. J Endovasc Ther 2011; 18: 613-623.

3. Suzuki K, Iida O, Soga Y, Hirano K, Inoue N, Uematsu M, et al. Long-term results of the S.M.A.R.T. Control ${ }^{\mathrm{TM}}$ stent for superficial femoral artery lesions, J-SMART registry. Circ J 2011; 75: 939-944.

4. Iida O, Soga Y, Hirano K, Okamoto S, Dohi T, Uematsu M, et al. Retrospective multicentre analysis of S.M.A.R.T. vs. Luminexx nitinol stent implantation for superficial femoral artery lesions (REAL SL) Registry: 5 years' experience. Circ J 2011; 75: 421-427.

5. Awata M, Nanto S, Uematsu M, Morozumi T, Watanabe T, Onishi T, et al. Heterogeneous arterial healing in patients following paclitaxeleluting stent implantation: Comparison with sirolimus-eluting stents. JACC Cardiovasc Interv 2009; 2: 453-458.

6. Nakazawa G, Finn AV, Vorpahl M, Ladich ER, Kolodgie FD, Virmani R. Coronary responses and differential mechanisms of late stent thrombosis attributed to first-generation sirolimus- and paclitaxel-eluting stents. J Am Coll Cardiol 2011; 57: 390-398.

7. Finn AV, Joner M, Nakazawa G, Kolodgie F, Newell J, John MC, et al. Pathological correlates of late drug-eluting stent thrombosis: Strut coverage as a marker of endothelialization. Circulation 2007; 115: $2435-2441$. 